\title{
Theoretical Investigation by Quantum Mechanics on the Tunnel Diode Effect of Electric Conductive Characteristics and Haptic Sensing in MCF Rubber
}

\author{
Kunio Shimada \\ Faculty of Symbiotic Systems Science, Fukushima University, 1 Kanayagwa, Fukushima 960-1296, Japan \\ Correspondence should be addressed to Kunio Shimada, shimadakun@sss.fukushima-u.ac.jp
}

Received 20 August 2010; Accepted 2 November 2010

Academic Editor: Ignacio Matias

Copyright (C) 2010 Kunio Shimada. This is an open access article distributed under the Creative Commons Attribution License, which permits unrestricted use, distribution, and reproduction in any medium, provided the original work is properly cited.

\begin{abstract}
By applying our developed intelligent fluid, magnetic compound fluid (MCF), to silicon oil rubber, we have made the MCF rubber highly sensitive to temperature and electric conduction. MCF is useful as the element material in haptic robot sensors and other related devices. In the present paper, we clarified the relationship between the electric current and the voltage under a tensile strain by utilizing the quantum mechanics theory on the multibarrier potential problem. The experimental results could be qualitatively explained by our proposed theory. The electrons can be moved between the solid materials by the tunnel effect. The relation between voltage and electric current is affected by the formation of the clusters, and it is changed by the application of heat. We also clarified experimentally the present MCF rubber useful in haptic sensors. Because the motions of humans and robots are different, the sensing of the rubber is different, depending on the placement. However, as for both motions of human and robot, there is no quantitative difference in the electric resistance among kinetic energy, momentum, and force. The sensing is also different based on the stiffness of the surface to which the sensor is adhered.
\end{abstract}

\section{Introduction}

In the ongoing development of engineering technology, the development of new composite materials is needed in, for example, the fields of robotics, sensing, and related areas. In response to this need, Shimada has demonstrated experimentally the use of his intelligent fluid, magnetic compound fluid (MCF) [1-3]. MCF was created by compounding the nm-size magnetite particles $\left(\mathrm{Fe}_{3} \mathrm{O}_{4}\right)$ of magnetic fluid (MF) and $\mu \mathrm{m}$-size iron ( $\mathrm{Fe}$ ) particles into a single solvent, thereby essentially producing a mixture of MF and magnetorheological fluid (MRF). The apparent viscosity of MF under a magnetic field is lower than that of MRF. Therefore, the engineering applications utilizing MRF are more advantageous than those using MF. However, the stability of particles distribution in an MF solvent is greater than that in MRF. The merit of MF is that it behaves as a fluid, because it does not have the problem of particle deposits like MRF does. In contrast to MF and MRF, MCF has the respective merits of both MF and MRF. The apparent viscosity of MCF is larger than that of MF, and the particles stability of MCF is better than that of MRF. In addition, the apparent viscosity of MCF can be changed by adjusting the composition of the magnetic particles of $\mathrm{Fe}_{3} \mathrm{O}_{4}$ and $\mathrm{Fe}$ and solvent as discussed by Shimada et al. [1-3] and by Fujita and Shimada [4]. Therefore, we can control the various characteristics of MCF, because Shimada succeeded in extracting the magnetic clusters formed by the magnetic particles from both MCF and MRF [5]. Since we have come to understand the behavior of the magnetic clusters, MCF has become a candidate for many engineering applications as discussed by Shimada et al. [6]. For example, MCF has been used effectively as a damper in the fields of architecture and vibrating machines as discussed by Shimada et al. [7] and by Kanno et al. $[8,9]$ and for magnetic fluid polishing by as discussed $\mathrm{Wu}$ et al. [10] and by Shimada et al. [11, 12]. These damping and polishing effects are enhanced by the magnetic clusters in the MCF, and the effects are greater than those with ordinary MF or MRF dampers and ordinary MF or MRF polishing. 
As another application utilizing the MCF, rubber with magnetic clusters is a new composite material that responds to a magnetic field. By compounding MCF into a silicon-oil rubber, the magnetic clusters can be aligned in the rubber by drying the rubber under the application of a magnetic field as discussed by Shimada et al. [13]. MCF rubber has anisotropic magnetic and material dynamic characteristics and is capable of elastic motion. Thus, MCF rubber is useful for engineering applications, for example, as artificial skin for a robot. Because the greater tensile silicon-oil rubber is used in the MCF rubber, the application of the MCF rubber to the artificial skin is effective. The characteristics of the tension of the MCF rubber have been clarified by Shimada and Zheng $[14,15]$, as has the relation among electric resistance, Young's modulus, and temperature sensitivity in the MCF rubber. These characteristics make MCF rubber applicable as material for a robot's haptic sensors as discussed by Tanaka et al. [16] and by Tajima et al. [17]. A haptic sensor must have high electric and temperature sensitivity. By applying the MCF to a silicon oil rubber, we can make MCF rubber highly sensitive to temperature and electric conduction as discussed by Shimada and Zheng [18] and by Zheng and Shimada $[19,20]$. By mixing $\mathrm{Cu}$ and $\mathrm{Ni}$ particles in the silicon oil rubber and by applying a strong magnetic field to it, we can produce magnetic clusters at a high density. The clusters form a network, as the schematic clusters model confirmed by optical observation, and as shown in Figure 13(a). On the other hand, MCF rubber containing Fe particles forms needle-like magnetic clusters, as shown in Figure 13(b). A comparison between the cluster structures shows that the former, with its network-like clusters, has greater temperature and electric sensitivity than does the latter. Thus, by using network-like magnetic clusters in the MCF rubber, we can expect to make a haptic sensor with high electric and temperature sensitivity as discussed by Zheng and Shimada [21]. The electric conductivity of the MCF rubber that has network-like magnetic clusters is greater than that of ordinary commercial base electric conductive rubber. In the case of the MCF rubber, the electric sensitivity is lower at small deformations but increases at larger deformations, as shown in Figure 14. Therefore, MCF rubber is effective for switching sensors when a small deformation is applied to it. It has high electric conduction sensitivity. Furthermore, the MCF rubber having higher electric sensitivity was improved by condensing dotite A and $\mathrm{B}$ as electric conductivity paint with carbon blacks made by Namamura Rikagaku, Ltd., Co. in Japan as discussed by Zheng and Shimada [22]. The improved MCF rubber has high resistance to large changes induced by compression. The electric resistance of the improved MCF rubber is megaorder $\Omega$ without compression, and it becomes $335 \Omega$ under $0.294 \mathrm{~N}$ of compression. In general, less than $1 \mathrm{~N}$ is needed for a haptic sensor, for example, for artificial skin. The utilizing of carbon for higher electric resistivity by low force and small displacement is effective, and there are many studies utilizing carbon blacks as discussed by Pyne [23], carbon fiber as discussed by Wang and Chung [24], or carbon nanotubes as discussed by Cao et al. [25]. However, in rubber with these carbon materials, the electric resistance without compression is very small, and thus the change of the electric resistivity by compression is small. In contrast, the improved MCF rubber has greater electric resistivity change induced by compression for inducing carbon blacks, because the other metal particles are involved in the MCF rubber. These facts imply the difference between the MCF rubber and the ordinary commercial base electric conductive rubber. And they also suggest that the MCF rubber has advantage. As for the MCF rubber, the electric current does not flow at the case of touching the rubber without any compressed force; however, it flows at the case of touching it with large compressed force. Contrastively, as for the ordinary commercial base electric conductive rubber, the electric current flows at the case of touching the rubber without any compressed force, and it also flows at the case of touching it with large compressed force. And its change of the flowing electric current is small as compared with the one at the case of touching it without any compressed force. Therefore, the MCF rubber is more effective for switching than the ordinary commercial base electric conductive rubber. The switching effect is more useful for haptic robot sensors because of larger change of the flowing electric current at the case of applied large compressed force from the case of touching it without any compressed force. Except for the switching effect, the MCF rubber has more advantages as compared with the ordinary commercial base electric conductive rubber. The MCF rubber has anisotropic magnetic and material dynamic characteristics. The cause is due to the orientation of the magnetic clusters induced by the metal particles of the MCF rubber. Therefore, the mechanical motion and the sensitivity can be changed anisotropically along their orientation in the case of engineering applications in the field of robot. In addition, the MCF rubber is more elastic than the ordinary commercial base electric conductive rubber. The cause is due to the metal particles of $\mathrm{Cu}, \mathrm{Ni}, \mathrm{Fe}$, and so on involved in the MCF rubber. In the case of utilizing carbon nanotube in the silicon oil rubber, as an example of the ordinary commercial base electric conductive rubber, the rubber is very stiff, and it is not appropriate for the rubber to cover the robot since the robot cannot move easily. For the artificial skin of the robot, more elastic rubber is useful.

For an artificial skin for a robot and other engineering applications, a texture with high sensitivity is needed, and many textures have been proposed as discussed by Pruitt et al. [26], by Ausanio et al. [27], and by Maheshwari and Saraf [28]. However, the theoretical mechanism of electric conductivity in the rubber with metal particles, with large electric resistivity by compression, has not been clarified. Essentially, the electric current cannot transit between the metals because rubber exists between the metals. A new theoretical model should be proposed to explain the experimental phenomena.

Concerning the study of electric conductivity in the rubber, the conducting polymer is a well-known material whose electric characteristics have been investigated in many studies. A major focus of these studies is the development of electric conductivity with polymer or crystals as discussed by Van Roggen [29] and Sherman et al. [30] or with the conducting of silica or oxide materials as a doping carrier 


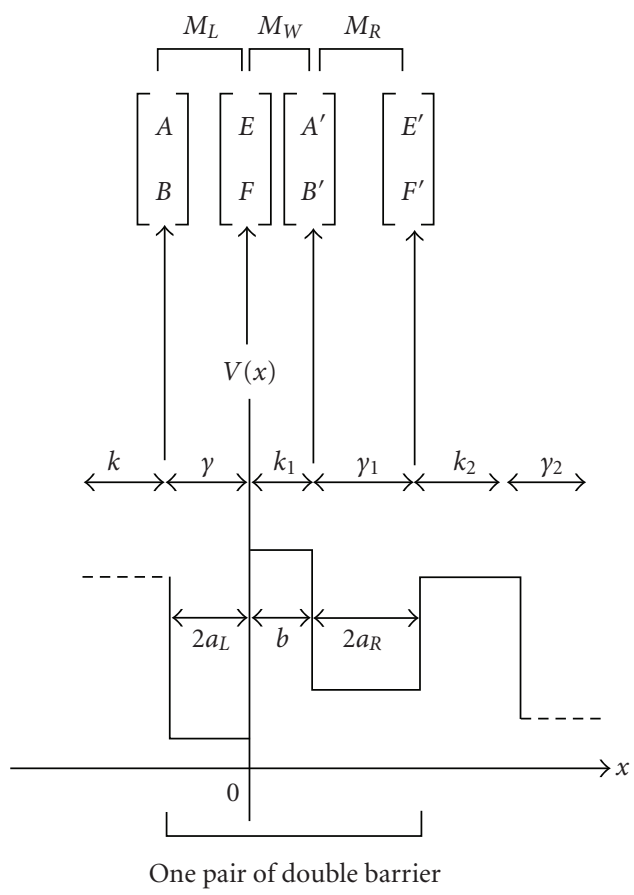

Figure 1: Model of the potential barrier in MCF rubber by quantum theory.

as discussed by Shimamura et al. [31], by Sichel et al. [32], by Sheng [33], by Nishio et al. [34], by Banerjee and Chakravorty [35], by Gangopadhyay et al. [36], by Bhattacharya and Ghosh [37], and by Van Roggen and Meijer [38]. However, there are few studies on the rubber type that we dealt with in the present study that focus on its ability to conduct metal particles as discussed by Shigeo et al. [39]. As for the electric characteristics of the ordinal electric conductive rubber, the electrical model has been proposed, but the theoretical explanation with calculation using quantum theory has not been constructed as discussed by Sherman et al. [30] and by Sheng [33], although the tunnel effect due to the electric conductivity was identified as well as the potential for the use of this rubber as semiconductor as discussed by Meijer and Van Roggen [40]. The present paper has the significant purpose of clarifying experimentally and theoretically the mechanism of electric conductivity in the rubber with metal particles with using quantum theory. In addition, the investigation of the sensing by the MCF rubber is also important. In the second half of the present paper, we investigate the characteristics of sensing during the motions of human and robot legs by setting MCF rubber on the bottom surfaces of a sole of the shoes and a robot's leg.

\section{Quantum Theory}

We examined the electron bond in the metal particles condensing in MCF rubber, $\mathrm{Fe}_{3} \mathrm{O}_{4}, \mathrm{Cu}, \mathrm{Ni}, \mathrm{Fe}$, and carbon blacks in dotite $\mathrm{A}$ and $\mathrm{B}$ and considered the behavior of the electrons transmitted over the potential barrier of the rubber

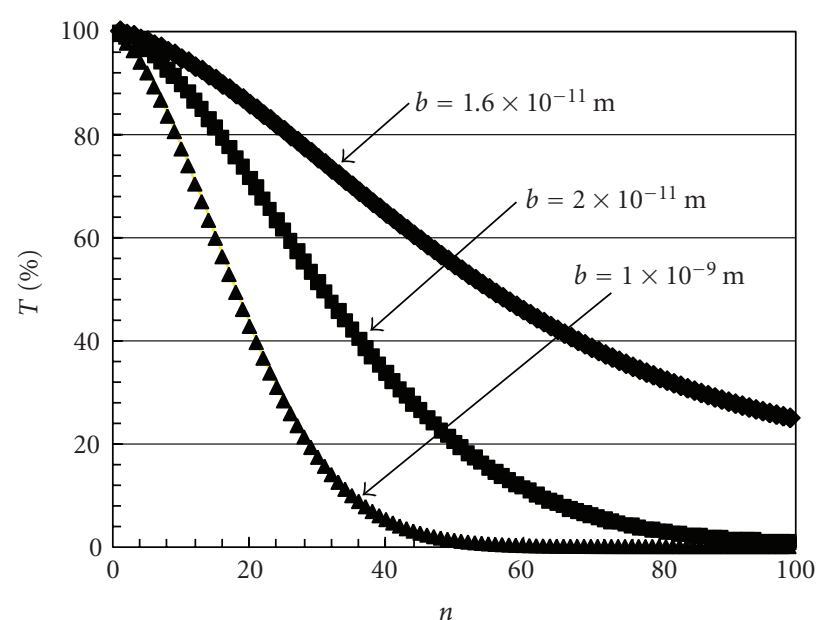

(a) Change of the transmission probability of electric current along thickness direction of MCF rubber

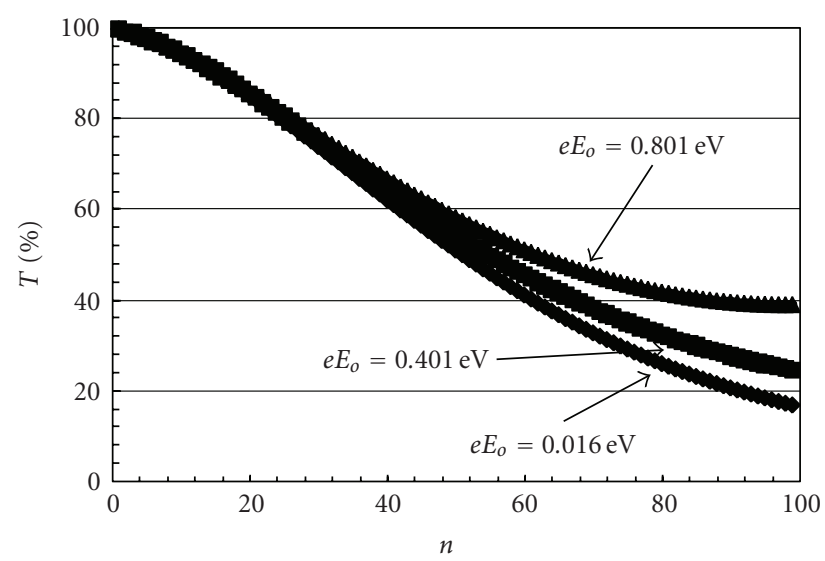

(b) Change of transmission probability of electric current to applied voltage

FIGURE 2: The change of the transmission probability along the MCF rubber's thickness direction.

as Schrödinger equation one-dimensionally in direction $x$, which is the direction of compression for the MCF rubber and of the transmitting electric current. We can deal with a pair of metal regions of $\gamma$ with a range of $2 a_{L}$ and of $\gamma_{1}$ with a range of $2 a_{R}$, and with one rubber region $k$ with a range of $b$ as the tunnel barrier problem on double barriers, and draw the diagram of potential energy $V$ as shown in Figure 1. MCF rubber is organized as $n$ pairs of these two metal and one rubber regions. Wave function $\Psi$ is presented by (1), where $\hbar$ is the $h / 2 \pi, h$ the Planck's constant, $m$ the mass of electron, $V_{o}$ the potential energy at regions $\gamma, \gamma_{1}$, or $k$, and $\varepsilon$ the energy of the electron:

$$
-\frac{\hbar^{2}}{2 m} \frac{\partial^{2} \Psi}{\partial x^{2}}+V_{o}(x) \Psi(x)=\varepsilon \Psi(x) .
$$

From (1), $\Psi$ is resolved as (2) and (3), respectively, for the regions $k$ and $\gamma$, where $A, B, C$, and $D$ are arbitrary constants that can be defined from the continuity of differential 


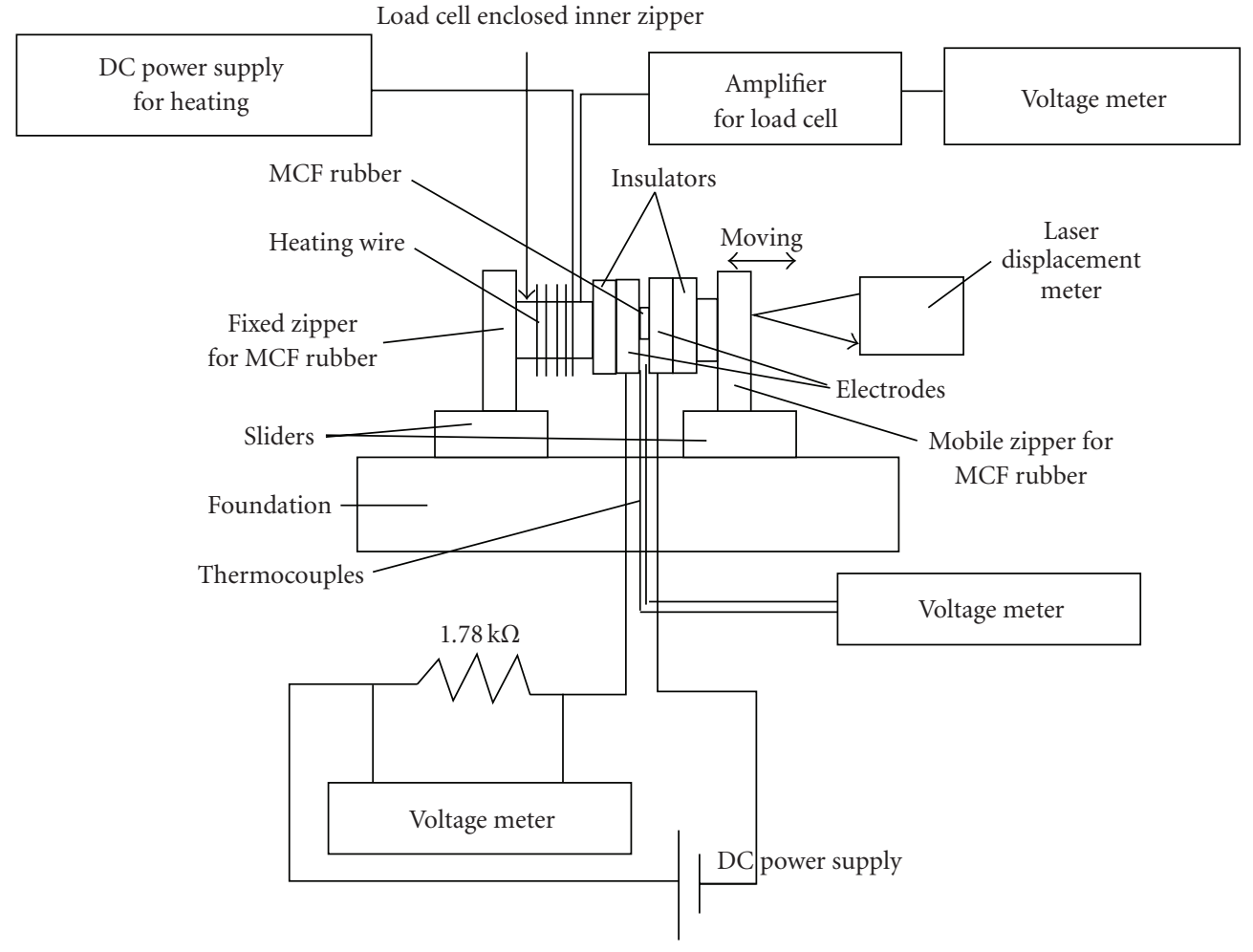

(a)

Detailed diagram of region around the MCF rubber settled between zippers.

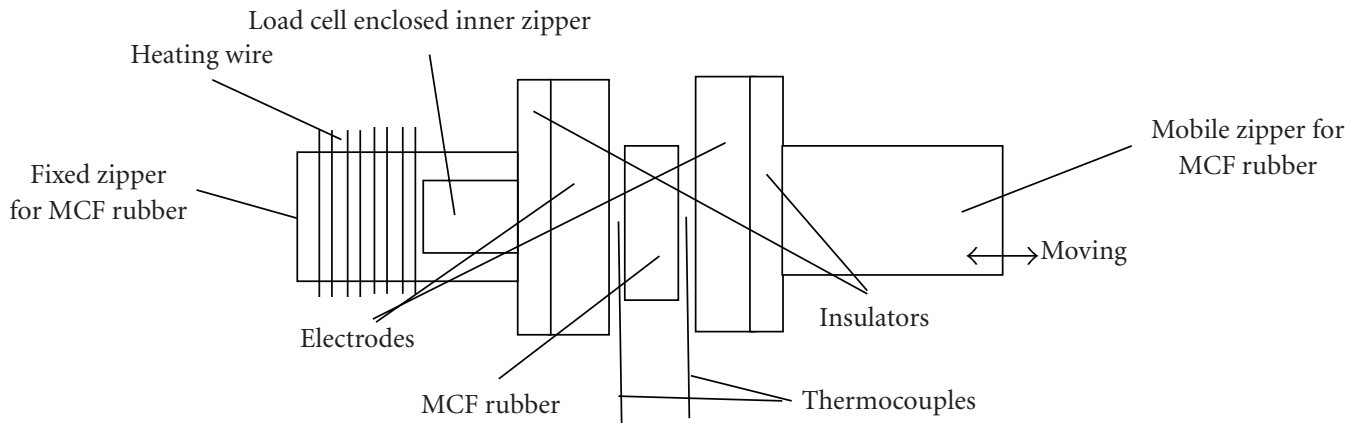

(b)

FIGURE 3: Schematic diagram of an experimental device.

coefficients on the boundary between $k$ and $\gamma\left(\gamma_{1}\right)$ regions as a well-known ordinary quantum problem, as follows:

$$
\begin{aligned}
& \Psi(x)=A e^{i k x}+B e^{-i k x}, \\
& \Psi(x)=C e^{\gamma x}+D e^{-\gamma x},
\end{aligned}
$$

where

$$
k=\sqrt{\frac{2 m}{\hbar^{2}} \varepsilon}, \quad \gamma=\sqrt{\frac{2 m}{\hbar^{2}}\left(V_{o}-\varepsilon\right)}
$$

On the one pair of double barriers shown in Figure 1, the arbitrary constants $A$ and $B$ exist in relation to constants $E^{\prime}$ and $F^{\prime}$ as shown in (5) as discussed by Van Roggen and Meijer [38], by Van Roggen and Meijer [41], and by Ferry [42], as follows:

$$
\left[\begin{array}{l}
A \\
B
\end{array}\right]=M_{L} M_{W} M_{R}\left[\begin{array}{l}
E^{\prime} \\
F^{\prime}
\end{array}\right]
$$

$$
M_{T}=M_{L} M_{W} M_{R},
$$




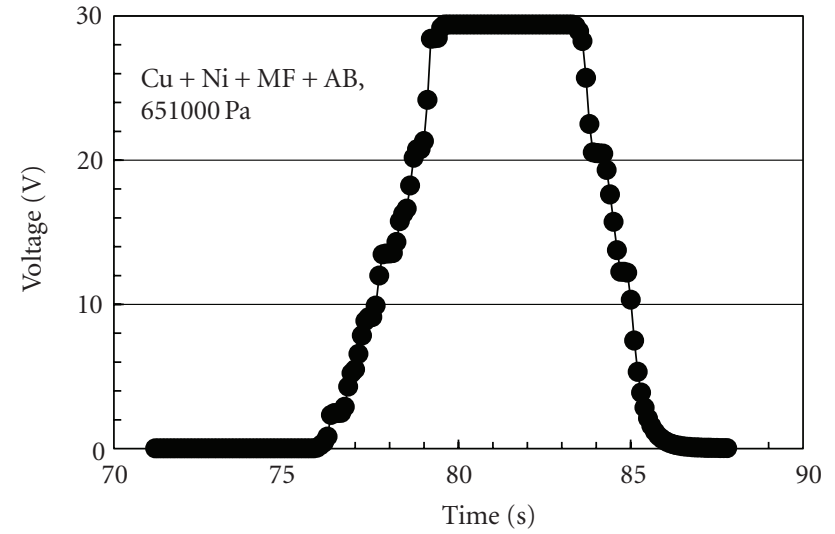

(a)

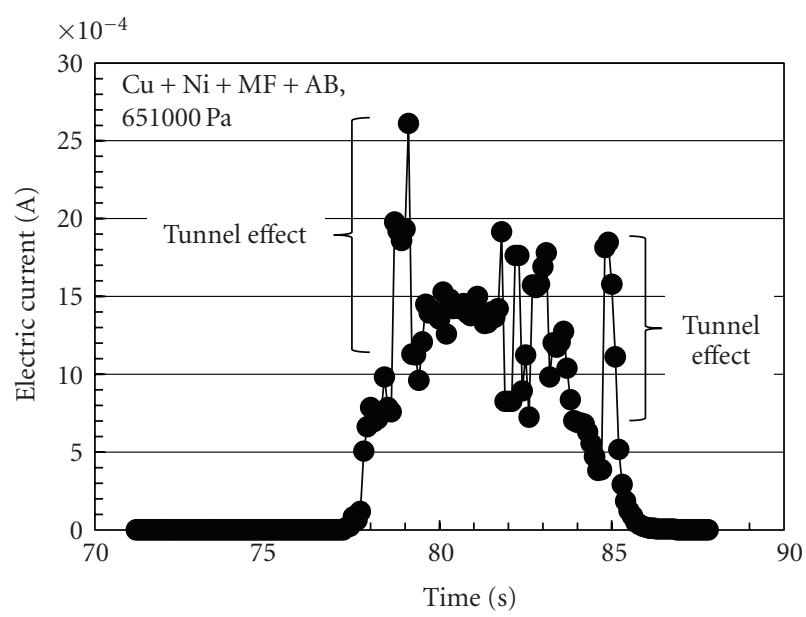

(b)

FIGURE 4: An example of the waveform of applied voltage and measured electric current under a constant pressure in the improved MCF rubber.

where

$$
\begin{aligned}
M_{L} & =\left[\begin{array}{cc}
m_{L 11} e^{i_{\theta_{L 11}}} & m_{L 12} e^{i_{\theta_{L 12}}} \\
m_{L 12} e^{-i_{\theta_{L 12}}} & m_{L 11} e^{-i_{\theta_{L 11}}}
\end{array}\right], \\
M_{W} & =\left[\begin{array}{cc}
e^{-i_{k_{1}^{b}}} & 0 \\
0 & e^{i_{k_{1}^{b}}}
\end{array}\right], \\
M_{R} & =\left[\begin{array}{ll}
m_{R 11} e^{i_{\theta_{R 11}}} & m_{R 21} e^{-i_{\theta_{R 21}}} \\
m_{R 21} e^{i_{\theta_{L 21}}} & m_{R 11} e^{-i_{\theta_{L 11}}}
\end{array}\right],
\end{aligned}
$$

$m_{L 11}$

$=\sqrt{\frac{1}{4}\left(1+\frac{k_{1}}{k}\right)^{2} \cosh ^{2}\left(2 \gamma a_{L}\right)+\frac{1}{4}\left(\frac{k k_{1}-\gamma^{2}}{k \gamma}\right)^{2} \sinh ^{2}\left(2 \gamma a_{L}\right)}$,

$m_{L 12}$

$$
=\sqrt{\frac{1}{4}\left(\frac{k k_{1}-\gamma^{2}}{k \gamma}\right)^{2} \sinh ^{2}\left(2 \gamma a_{L}\right)+\frac{1}{4}\left(\frac{k_{1}}{k}-1\right)^{2} \cosh ^{2}\left(2 \gamma a_{L}\right)},
$$

$m_{R 11}$

$=\sqrt{\frac{1}{4}\left(1+\frac{k_{2}}{k_{1}}\right)^{2} \cosh ^{2}\left(2 \gamma_{1} a_{R}\right)+\frac{1}{4}\left(\frac{k_{1} k_{2}-\gamma_{1}^{2}}{k_{1} \gamma_{1}}\right)^{2} \sinh ^{2}\left(2 \gamma_{1} a_{R}\right)}$,

$m_{R 21}$

$=\sqrt{\frac{1}{4}\left(\frac{k_{1} k_{2}-\gamma_{1}^{2}}{k_{1} \gamma_{1}}\right)^{2} \sinh ^{2}\left(2 \gamma_{1} a_{R}\right)+\frac{1}{4}\left(\frac{k_{2}}{k_{1}}-1\right)^{2} \cosh ^{2}\left(2 \gamma_{1} a_{R}\right)}$,

$\theta_{L 11}$

$=-\tan ^{-1}\left[\frac{k k_{1}-\gamma^{2}}{\left(k+k_{1}\right) \gamma} \tanh \left(2 \gamma a_{L}\right)\right]+\left(k+k_{1}\right) a_{L}$,

$\theta_{L 12}$

$=-\tan ^{-1}\left[\frac{k k_{1}+\gamma^{2}}{\left(k-k_{1}\right) \gamma} \tanh \left(2 \gamma a_{L}\right)\right]+\pi+\left(k-k_{1}\right) a_{L}$,

$\theta_{R 11}$

$=-\tan ^{-1}\left[\frac{k_{1} k_{2}-\gamma_{1}^{2}}{\left(k_{1}+k_{2}\right) \gamma_{1}} \tanh \left(2 \gamma_{1} a_{R}\right)\right]-\left(k_{1}+k_{2}\right) a_{R}$,

$\theta_{R 21}$

$=\tan ^{-1}\left[\frac{k_{1} k_{2}+\gamma_{1}^{2}}{\left(k_{2}-k_{1}\right) \gamma_{1}} \tanh \left(2 \gamma_{1} a_{R}\right)\right]+\pi+\left(k_{1}-k_{2}\right) a_{R}$.

The transmitted probability can be given by

$$
T=\left|M_{T 11}\right|^{2} .
$$

As the present MCF rubber has a lot of metal particles, one pair of double barriers is expanded to $n$ pairs of double barriers as multibarriers. Therefore, the matrix as shown by (5) is substituted into the matrix of the neighboring pair of double barriers, and this procedure is repeated to obtain the matrix of $n$ pairs of double barriers, where (9) can be given because the material of each region of $k$ and $\gamma$ is the same as that of the other regions. Here, $2 a$ is the thickness of metal particles, and $b$ is the thickness of rubber between the metal particles. When $e E_{o}$ is the applied voltage, the voltage $e E$ at the regions of $\gamma$ and $k$ is given as (9). Therefore, we calculate (7) as substituting $V_{o}-\varepsilon-e E_{o}$ for $V_{o}-\varepsilon$ of $\gamma$ in (4):

$$
\begin{gathered}
k=k_{1}=k_{2}=\cdots, \\
\gamma=\gamma_{1}=\gamma_{2}=\cdots, \\
a_{L}=a_{R}=a,
\end{gathered}
$$

$\frac{e E=e E_{o}\{(2 n-1) a+2(n-1) b\}}{L^{\prime}}$ at region of $\gamma$ before $k$, $\frac{e E=e E_{o}\{2 n a+(2 n-1) b\}}{L^{\prime}}$ at region of $\gamma$ after $k$. 


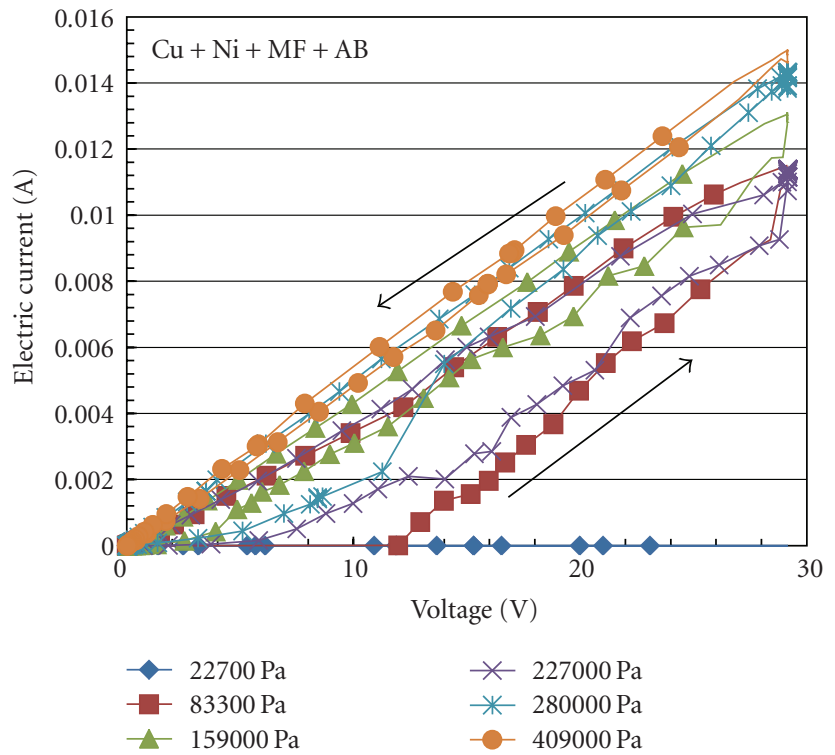

(a) In the case of improved MCF rubber

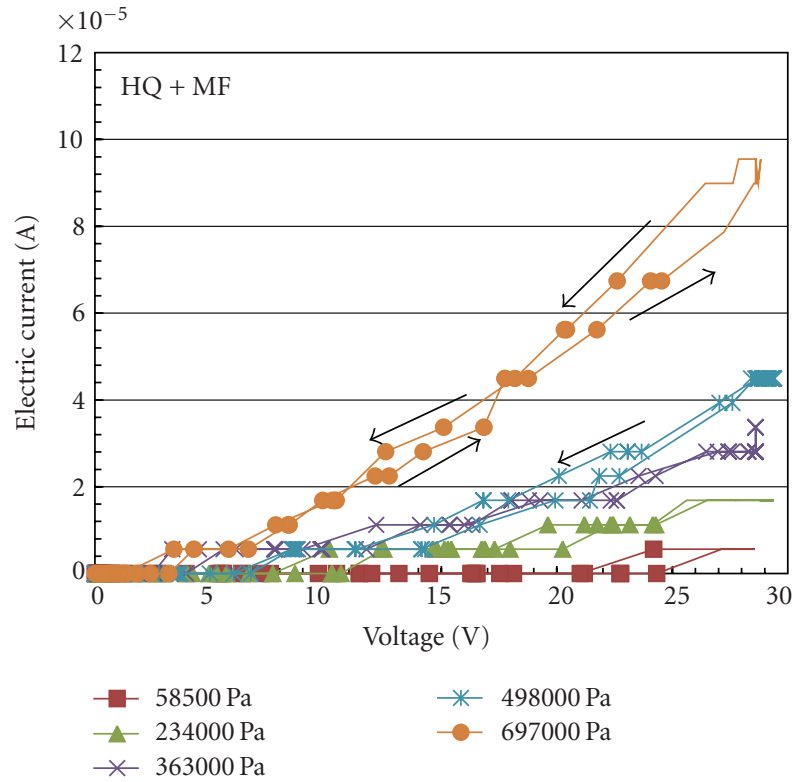

(b) In the case of MCF rubber with Fe particles

FIgURE 5: Relation between the electric current and the applied voltage.

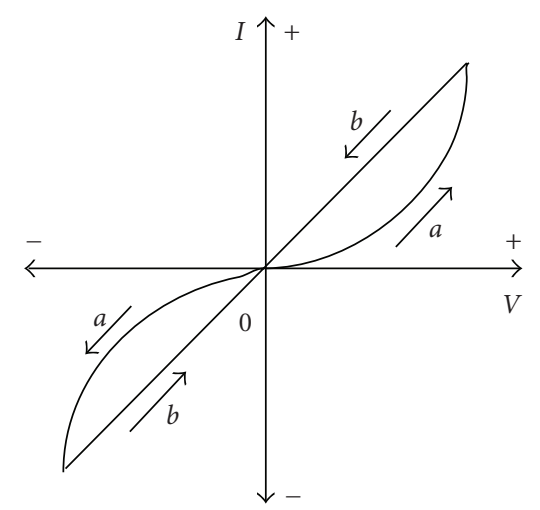

(a) Experimental data showing the relationship between the electric current and the applied voltage

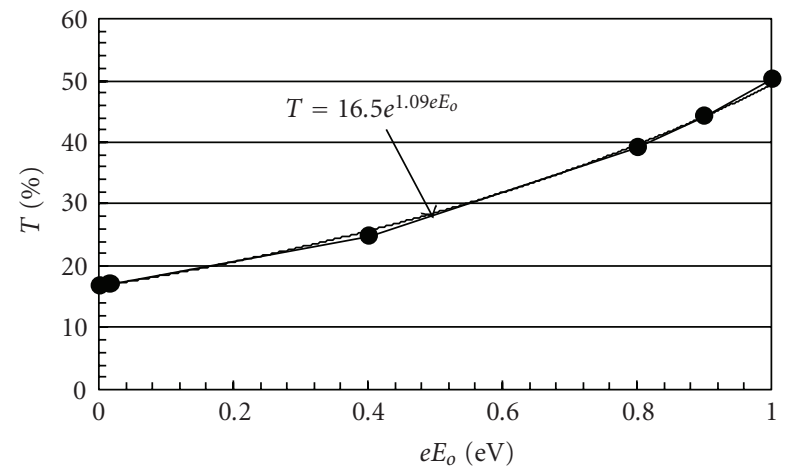

(b) Theoretical results of the relationship between the electric current and the applied voltage

FIGURE 6: Tendency by experiment and theory of the relationship between the electric current and the voltage in improved MCF rubber.
As the thickness of the MCF rubber is divided into $n$ pairs, the thickness of the MCF rubber $L^{\prime}$ can be given by

$$
L^{\prime}=n \Delta L=2 n(a+b)[\mathrm{m}] .
$$

Figure 2(a) shows the change of the transmitted probability $T$ along the points of $n$, that is, along the MCF rubber thickness direction. Along the horizontal axis, the electrons are transmitted from the left side to the right side. At this time, $T$ is decreasing, where the data is calculated under $V_{o}=4.98 \mathrm{eV}, \varepsilon=1.6 \mathrm{eV}, e E_{o}=0.401 \mathrm{eV}, a=10^{-12} \mathrm{~m}$. As the distance of the rubber $b$ is smaller, which means the MCF rubber is compressed, $T$ becomes larger. Therefore, it can be concluded that the cause of conduction of the electric current is the tunnel diode effect. The present theory demonstrates a series of our experimental results that the electric resistance is decreased by the compression of the MCF rubber. The change of $T$ to the applied voltage $e E_{o}$ is shown in Figure 2(b), where the data is calculated under $V_{o}=4.98 \mathrm{eV}, \varepsilon=1.6 \mathrm{eV}, a_{L}=a_{R}=10^{-12} \mathrm{~m}, b=$ $1.6 \times 10^{-11} \mathrm{~m}$. As $e E_{o}$ is increasing, the electric current can be transmitted easily.

\section{Verification Experiment}

We next verified the theoretical results experimentally. We used the experimental apparatus shown in Figure 3. A load cell (LSM-50K-B, by Minebea Co., Ltd., Japan) for measuring the compressed force was enclosed by an inner metallic zipper settled on the slider. This slider was fixed. The zipper was settled at the surface of the nonelectric conductive insulator that was in contact with a pair of metallic electrodes. At the opposite surface of the electrode, the MCF rubber was contacted. By sliding a second slider, the 


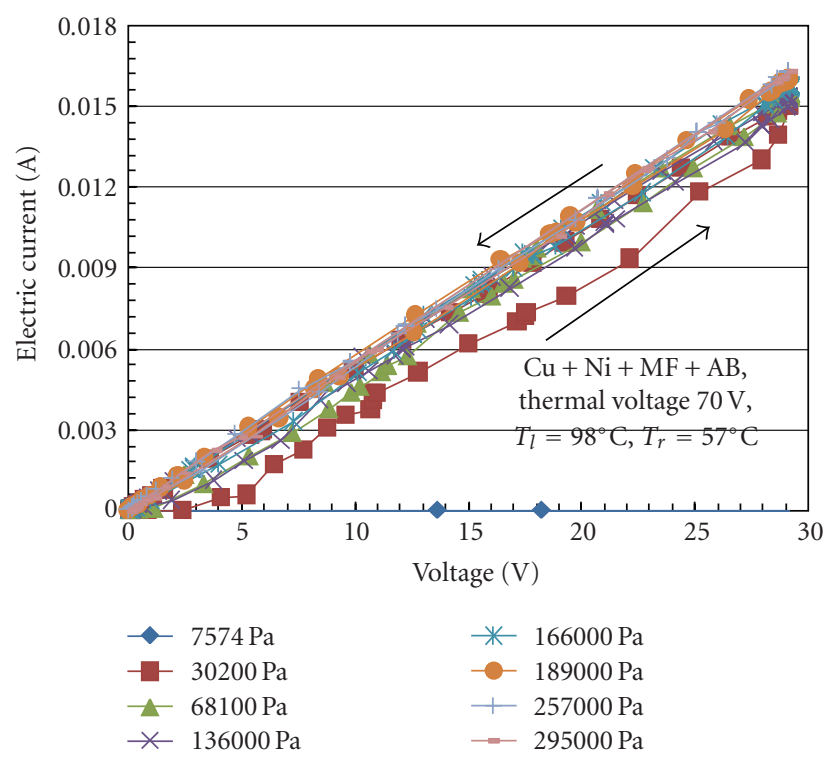

FIGURE 7: Relationship between the electric current and voltage in improved MCF rubber with the application of heat.

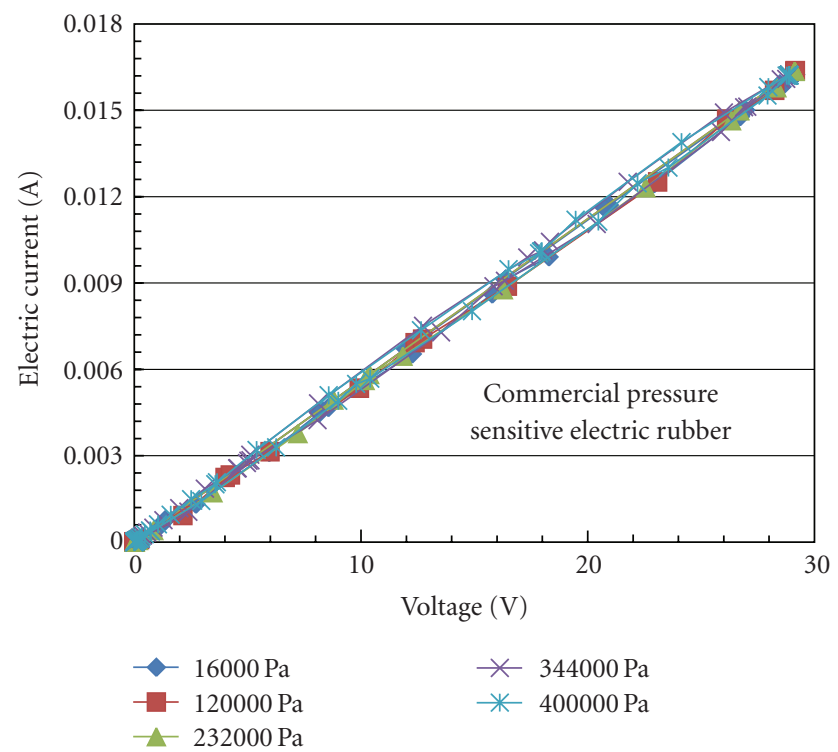

FIGURE 8: Relationship between the electric current and the applied voltage on commercial pressure sensitive electric rubber.

opposite surface of the MCF rubber was touched to another pair of metallic electrodes that was in contact with the other nonelectric conductive insulator. This electrode could be moved on the slider. The MCF rubber was compressed to make the electrodes move. At this time, the displacement was measured by a laser instrument (LK-G3000V, Keyence Co., Ltd., in Japan). In the present study, under a constant compressed force to the MCF rubber, electric current was measured when a voltage was applied to the electrodes.

We used MCF rubber samples that were hardened by lying between nonmagnetic plates with a thickness of $1 \mathrm{~mm}$ and under the application of permanent neodymium magnets with a surface magnetic flux density of $0.56 \mathrm{~T}$. The sample composites were $\mathrm{Ni}: \mathrm{Cu}: \mathrm{MF}$ (HS-50, kerosene base with $50 \mathrm{wt} \%$, Taiho Industrial Co. Ltd., Japan) : dotite A : dotite B : silicon oil rubber (SH9550, Toray-dow-corning Co., Ltd., Japan) = 1:1:1:1:1:10 to make improved MCF rubber with $11.5 \mathrm{~mm} \times 16.2 \mathrm{~mm} \times 0.855 \mathrm{~mm}$, and HQ (carbonil Fe particles, BASF Co., Ltd., Germany) : MF : SH9550 = $6: 4: 10$ with $13.1 \mathrm{~mm} \times 18.4 \mathrm{~mm} \times 0.5 \mathrm{~mm}$. We confirmed that the experimental results of the improved MCF rubber are the same as those of the MCF rubber without dotite in the present experiment. Therefore, we show here the results of the MCF rubber with dotite.

A fixed compressed force could not be applied by the apparatus as shown in Figure 3. Therefore, in the following figures, the quantitative values of the compressed force of each test are different. However, if you want to know the quantitative tendency at some value which is not indicated in the figures and which is between the values indicated in the figures, you can analogize from the tendency in the figures. This way was confirmed to be correct from many examinations.

Figure 4 shows an example of the experimental data of waveforms of the applied voltage and the measured electric current on the improved MCF rubber under a constant compressed force. We can sometimes confirm peaks in the electric current's waveform. A few peaks imply the tunnel effect, whose phenomenon is the momentary transmission of the electric current. Once electric current transmits between the metal particles, the route of the transmission of the electric current is up, and the electric current can be conducted easily, and then the waveform of the electric current is similar to that of the voltage. The peaks in the electric current's waveform do not necessarily appear clearly. However, it is incorrect to estimate that there is not tunnel effect because of the peaks not existing in the electric current's waveform. The peak in the electric current's waveform implies that the quantitative transmitted electric current between the metal particles is large. Regardless of the quantitative peak in the electric current's waveform, the cause that the electric current flows at the application of the voltage is due to the tunnel effect. Figures 5, 7, and 8 are the cases of small peak in the electric current's waveform.

The experimental results of having electric current at the indicated voltage under a constant compressed force in the case of improved MCF rubber are shown in Figure 5(a), and those in the case of MCF rubber with Fe are shown in Figure 5(b). The arrow in the figure indicates the direction of the setting turn of voltage from zero to maximum and then from maximum to zero.

As seen in Figure 5(a), the change of the electric current at the case of increasing voltage is qualitatively different from that at the case of decreasing voltage. The resulting qualitative tendency can be shown in Figure 6(a). The arrow in the figure means the direction of the setting turn of voltage from zero to maximum and then from maximum to zero. Figure 6(a) also shows the case of exchanging the electric current by reversing the electric poles. In Figure 5(a), we did not show the experimental results at the case of exchanging the electric current, because the same experimental results 


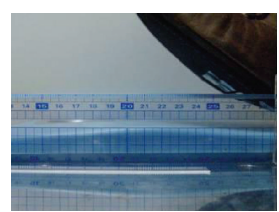

(i) $t=0 \mathrm{~s}$

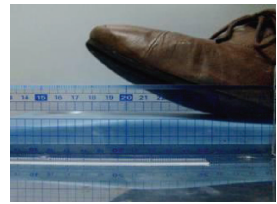

(iv) $t=0.3 \mathrm{~s}$

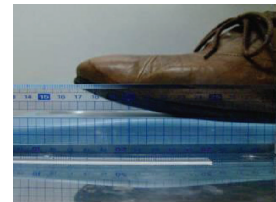

(vii) $t=0.6 \mathrm{~s}$

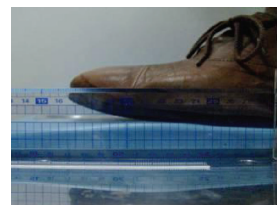

(x) $t=0.9 \mathrm{~s}$

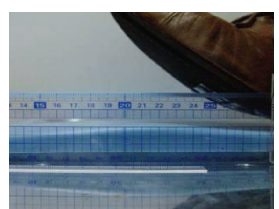

(ii) $t=0.1 \mathrm{~s}$

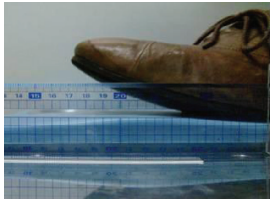

(v) $t=0.4 \mathrm{~s}$

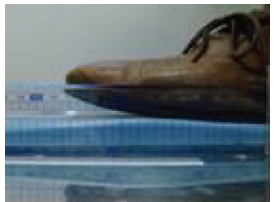

(viii) $t=0.7 \mathrm{~s}$

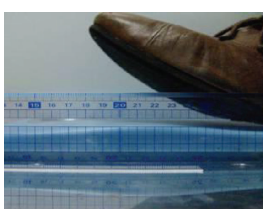

(iii) $t=0.2 \mathrm{~s}$

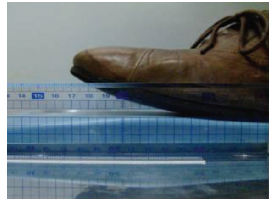

(vi) $t=0.5 \mathrm{~s}$

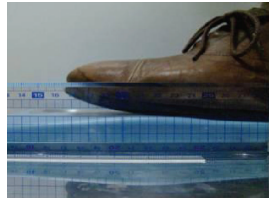

(ix) $t=0.8 \mathrm{~s}$

(a) In the case of the sole of the shoes with the improved MCF rubber

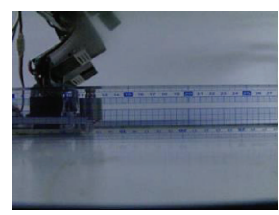

(i) $t=0 \mathrm{~s}$

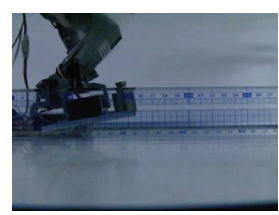

(iv) $t=0.09 \mathrm{~s}$

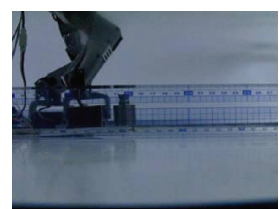

(vii) $t=0.19 \mathrm{~s}$

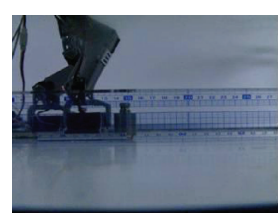

(x) $t=0.29 \mathrm{~s}$

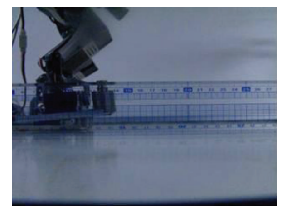

(ii) $t=0.03 \mathrm{~s}$

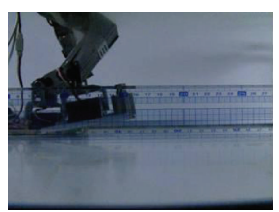

(v) $t=0.13 \mathrm{~s}$

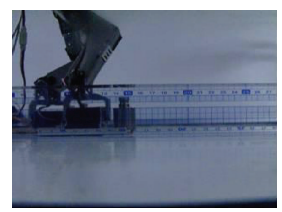

(viii) $t=0.23 \mathrm{~s}$

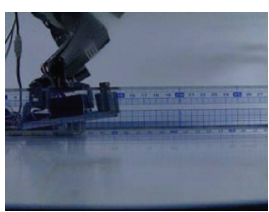

(iii) $t=0.06 \mathrm{~s}$

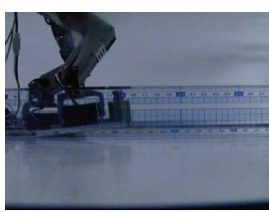

(vi) $t=0.16 \mathrm{~s}$

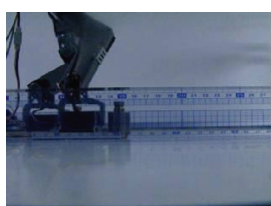

(ix) $t=0.26 \mathrm{~s}$

(b) In the case of the robot with the improved MCF rubber

Figure 9: Photographs of the moment of touching the ground. 


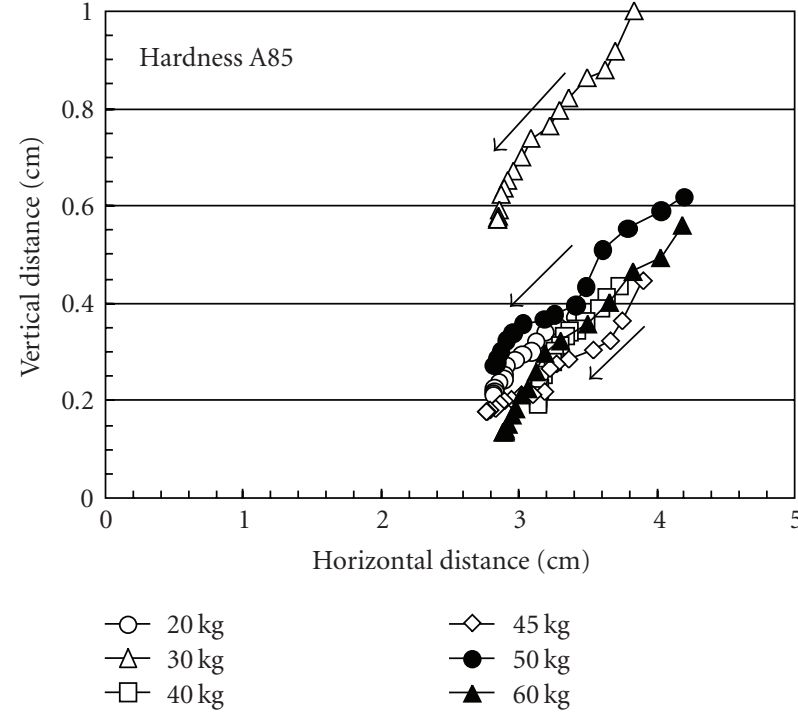

(a) In the case of the sole of the shoes

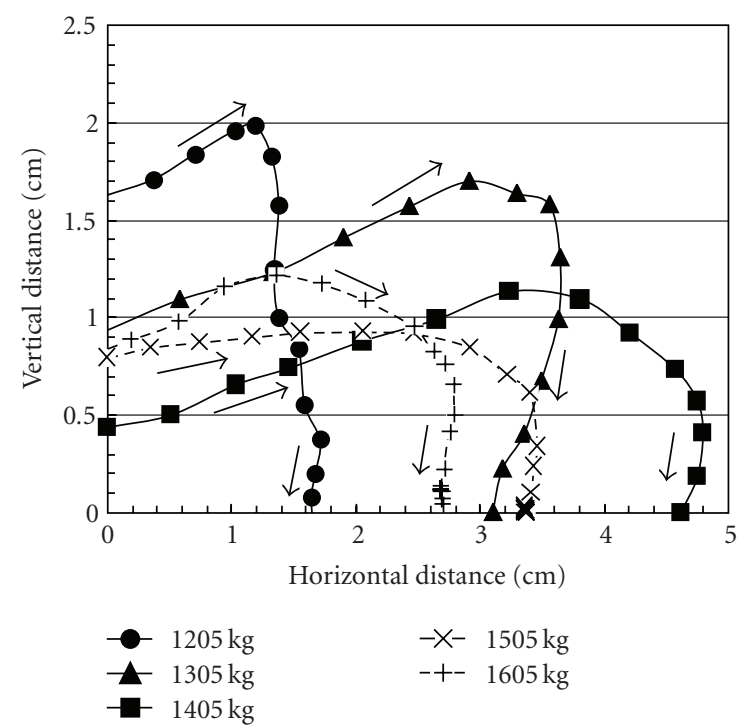

(b) In the case of the robot

Figure 10: The horizontal and vertical positions of the sole of the shoes and the robot leg during motion.

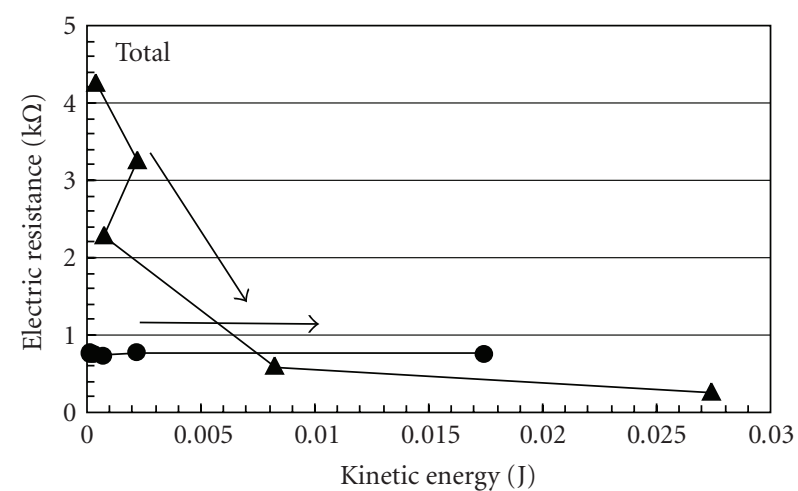

Hardness A65

- Hardness A85

(a)

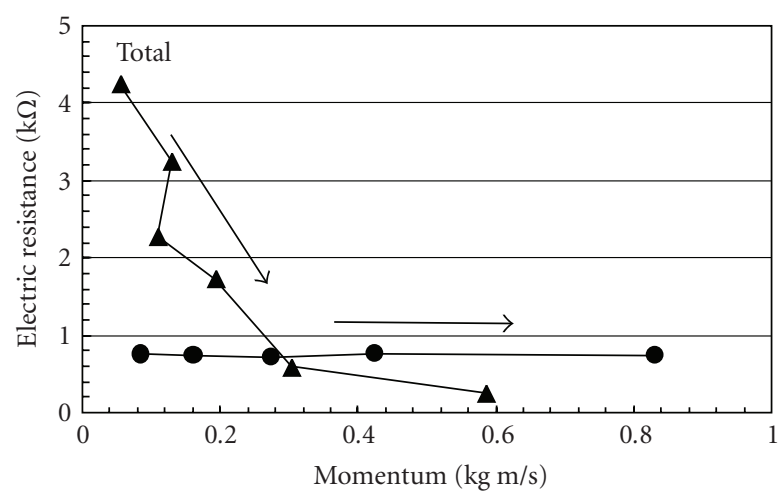

Hardness A65

Hardness A85

(b)

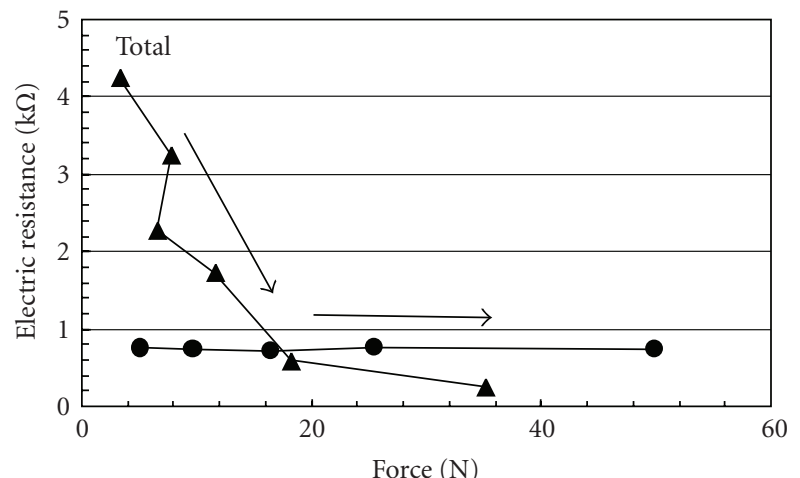

- Hardness A65

- Hardness A85

(c)

FIGURE 11: Change of electric resistance to kinetic energy, momentum, and force in the case of the sole of the shoes. 


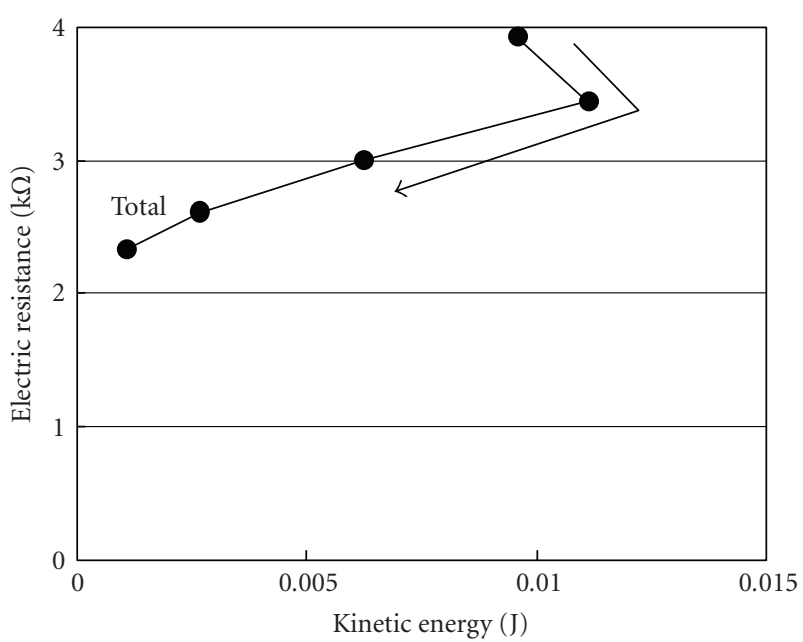

(a)

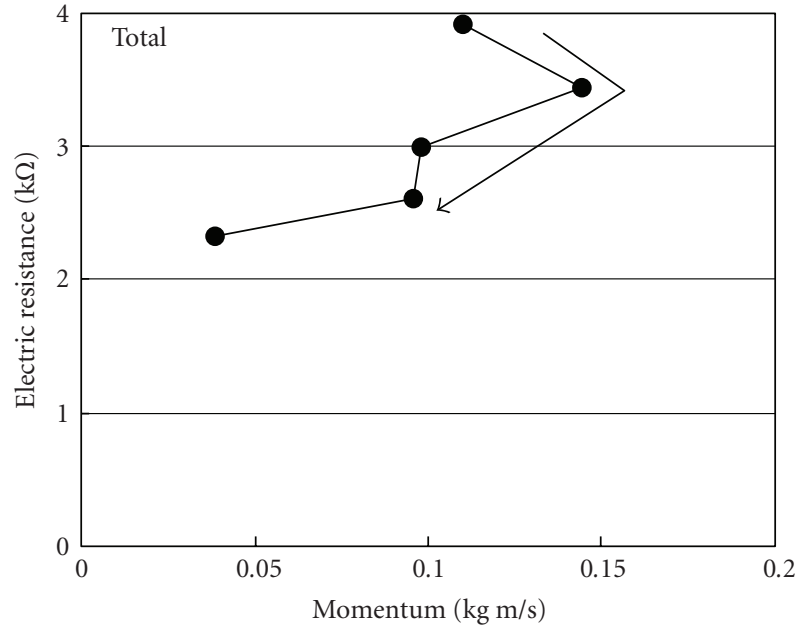

(b)

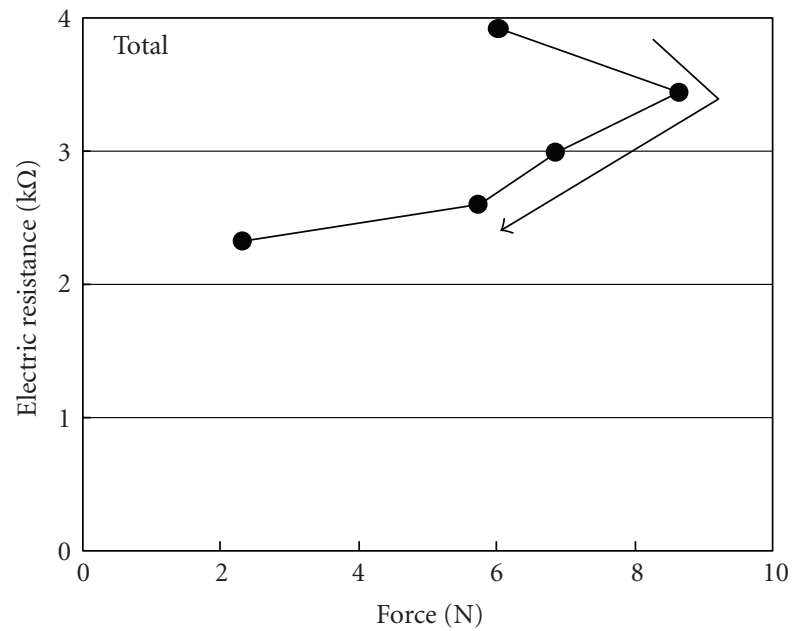

(c)

Figure 12: Change of electric resistance to kinetic energy, momentum, and force in the case of the robot.

as in Figure 5(a) were obtained. The tendency of line A in Figure 6(a) for the electric current to be enhanced gradually with increasing voltage means that the electric resistance is large, because the distance between the metal particles performs as the well-known ordinary space layer in the case of a semiconductor.

Once electric current is transmitted between the metal particles, the route of the transmission of the electric current is up, the electric current can be conducted easily, and the electric resistance becomes constant, and then the electric current takes on line $\mathrm{b}$ in Figure 6(a). Therefore, when the applied voltage begins decreasing, the tendency of the relation between the voltage and the electric current becomes linear, as shown in line b in Figure 6(a).

The tendency of line a in Figure 6(a) can be explained by the present quantum theory. $T$ is calculated by the equations in the previous section under $V_{o}=4.98 \mathrm{eV}, \varepsilon=1.6 \mathrm{eV}$, $a_{L}=a_{R}=10^{-12} \mathrm{~m}$, and $b=1.6 \times 10^{-11} \mathrm{~m}$, as shown in Figure 6(b). With increasing applied voltage, the electric current increases gradually. The tendency is nonlinear.
Here, the point to be noted is that the tendency in the case of reversing the electric poles is the same as that in the case of before changing them. This is different from the case of a well-known ordinary semiconductor. The cause is due to the independence of the direction of the electric current in the case of the rubber with metal particles.

As the compressed force grows larger, because the transmitted electric current is enhanced, the relation between the voltage and the electric current converges to the linear relation shown in line $b$ in Figure 6(a). The experimental results are coincident with the theoretical ones shown in Figure 2(a).

Next, we compare Figures 5(a) and 5(b). The latter shows the tendency that the relation between the voltage and the electric current becomes close to linear. The cause is that the electric current can more easily pass through the straight line like that of the needle-shaped clusters than through the winding line like that of the network-shaped clusters. Therefore, the shape of the clusters influences the relation between the voltage and the electric current. 


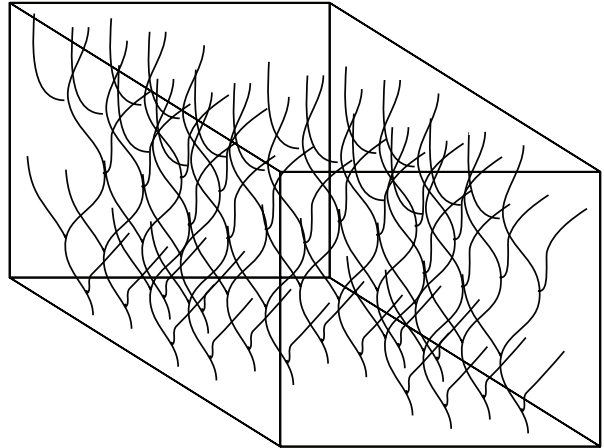

(a) Using $\mathrm{Ni}$ and $\mathrm{Cu}$

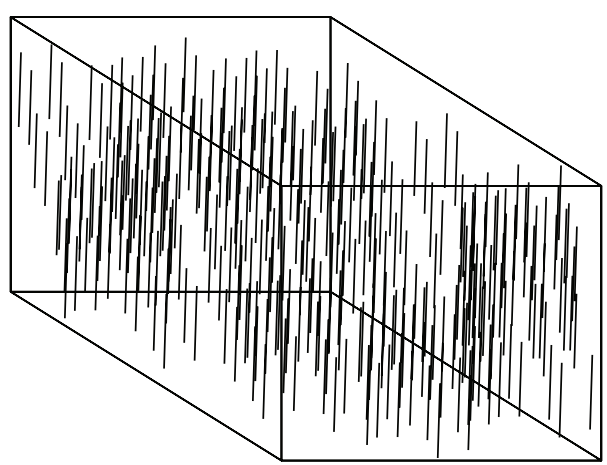

(b) Using Fe

FIgURE 13: Model of magnetic clusters in MCF rubber as shown in the previous studies [18-21].

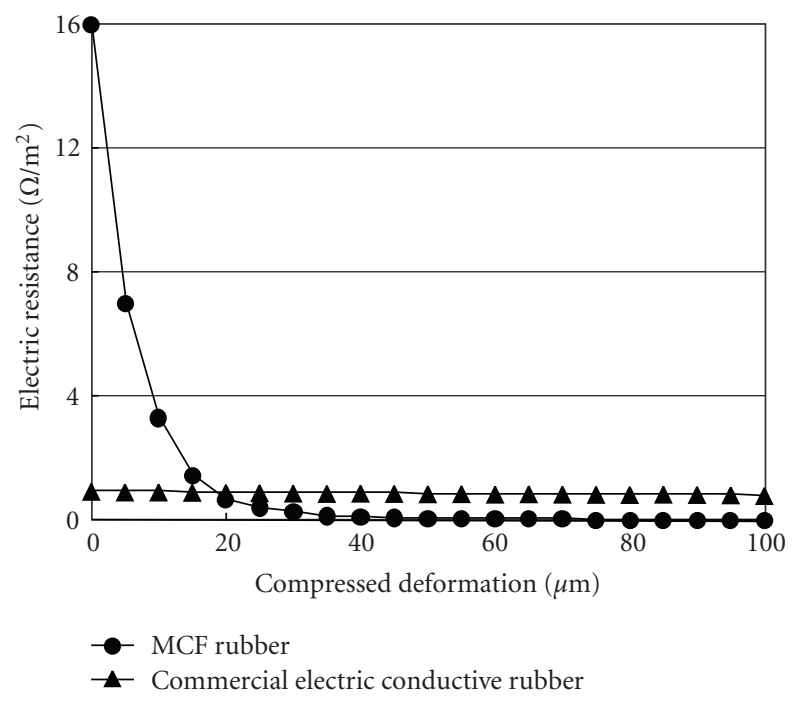

FIGURE 14: Comparison of electric resistance to compressed deformation between MCF rubber and commercial electric conductive rubber.

Next, as we investigate the effect of heat on the transmission of the electric current, we compare Figures 5(a), 7. Figure 7 shows the relation between electric current and voltage in the improved MCF rubber with the application of heat. As shown in Figure 3, the heater with the shape of wire is wound around the fixed zipper for the MCF rubber, and thermocouples are inserted between the electrodes and the MCF rubber. While the temperature of the thermocouples was held constant without applying any compressed force, the electric current to the voltage was measured. The temperature, $T_{l}$, is that of the MCF surface on the side being heated, and $T_{r}$ is the temperature of the MCF surface on the side where heat conducting is occurring. $T_{l}$ and $T_{r}$ in Figure 7 are the constant temperatures before applying the compressed force to the MCF rubber. By heating, the electrons can be transmitted between the metal particles, because the thermal energy raises the potential energy. Therefore, as the electric resistance decreases, the relation between the electric current and the voltage becomes linear.
Finally, we compare the present MCF rubber to the ordinary commercial base electric conductive rubber, as shown in Figure 8. As the commercial rubber (made by Yokohama Rubber Co., Ltd., Japan) has carbon nanotubes, the electric current can be conducted through it without any pressure over the rubber surface, including in the rubber's thickness direction. Therefore, the quantitative change of the electric current by applying compressed force under the applied constant voltage is smaller than that of the present MCF, as shown in Figure 14. In addition, the tunnel effect does not occur, and then the relation between electric current and voltage becomes linear. Therefore, the MCF rubber is effective for switching sensors when a small deformation is applied.

\section{Insights about Sensing}

As for the present MCF rubber, the investigation of the sensing characteristics of the rubber settled on a human body or robot is important. There are many studies about the sensing characteristics of haptic sensors settled on the human hand or machine as discussed by Tanaka et al. [43], by Tajima et al. [44], and by Shimizu [45]. However, the characteristics of sensitivity to the motions of the human body and robots have not been investigated in detail. Since haptic sensors can be settled on human and robot bodies, we must investigate their sensing characteristics in detail. The difference in the motion between human body and robots influences the sensing characteristics, because there are differences in the horizontal and vertical forces on the touched surface between human body and robots. And the difference in the stiffness of the adhered surface by the haptic sensors also influences the sensing characteristics. We should investigate these differences by utilizing the present highly sensitive improved MCF rubber, as shown in Figure 5(a). As we compared the characteristics between the human body and a robot, we dealt with the leg motion. The MCF rubber was settled on each bottom surface of a sole of shoes and a robot's leg. Their hardness was different. As for the former, we used two types of the hardness. They were measured as A85 and A65 according to the Japanese industrial standardization of JIS K 6253 by using 
a hardness meter (WR-202NA, Nishi-Tokyo-Semimitsu Co., Ltd., Japan), and the latter was A100. For reference, as for human body, it is A10. Electrodes were also adhered to the surfaces of two sides of the MCF rubber, and the electric current was measured by applying voltage to the electrodes. A human subject and a robot walked on a hard ground surface. As for the sole, the maximum force when touching the ground was measured by a weight scale. As for the robot, a measured weight was put on the robot's leg. At the instant the sole or leg touched the ground, the electric current was measured and the electric resistance calculated. Our robot is the KHR-2HV, made by Kondo Kagaku Co., Ltd., Japan. The robot can walk by controlling touch softly on the ground surface with feedback fuzzy control. Figure 9 shows each motion of the sole of the shoes and the robot leg when touching the hard ground. Analyzed from the photographs of Figure 9, the motions divided into horizontal and vertical positions are shown in Figure 10. The arrows in the figures indicate the motion direction. In the case of the sole of the shoes, the MCF rubber strikes the ground moving straight down. However, in the case of the robot, the MCF rubber does not strike the ground moving straight down because of the soft touch by feedback fuzzy-controlling force adjusted from the control system of the robot. In general, in the field of the robot, feedback fuzzy-controlling is always used for the motion. We can suppose that the haptic sensor can be used in the human body or the robot. The former is related to the motion of human and the latter to the motion of robot as shown in Figures 9 and 10. In the present paper, we investigate the difference of the haptic senor's characteristics between both motions.

The velocity and acceleration can be obtained by analyzing Figures 9 and 10, giving us Figures 11 and 12, respectively. The arrows in the figures indicate the motion direction. There is no quantitative difference in the electric resistance among the kinetic energy, momentum, and force. The kinetic energy and the momentum are related to the velocity. The force is related to the acceleration. Therefore, there is also no quantitative difference in the electric resistance among the velocity and the acceleration. On the other hand, with the lesser hardness, the change of the electric resistance becomes constant by the kinetic energy, momentum, and force. Therefore, sensing the stiffness of the surface is important for the sensor adhered to the robot's leg. In the case of the sole of the shoes, since the MCF rubber strikes the ground straight down, the kinetic energy, momentum, and force are increased, and the electric resistance becomes smaller gradually. However, in the case of the robot, where the MCF rubber does not strike the ground straight down because of the soft touch by feedback fuzzy-control, the change of the electric resistance is not gradual as increasing the kinetic energy, momentum, and force, as shown by the arrow shown in Figure 12.

\section{Conclusion}

The relation between the electric current and the applied voltage on a rubber sample with network-like clusters of metal particles is non-linear at the time of applying the increasing voltage but linear at the time of applying the decreasing voltage. The former tendency occurs because the electrons cannot easily transit between the metal particles. The experimental tendency can be explained by the tunnel effect on quantum mechanics. As the electric current can more easily pass through a straight line like the needle shape of the clusters than through a winding line like the network shape of the clusters, the relation between the applied voltage and the electric current is dependent on the clusters' shape. By applying the heat, the electric current can easily transit between the metal particles.

The present improved MCF rubber is useful for haptic sensors. By testing the difference in feedback between the motions of a human leg and a robot leg, we have demonstrated that the sensing of the rubber is different. There is no quantitative difference in the electric resistance among the kinetic energy, momentum, and force. The sensing is also different according to the stiffness of the surface to which the sensor is adhered.

\section{Appendix}

Figure 13 shows the cluster model formed in the MCF rubber by the particles. In Figure 13(a), the particles in the MCF rubber are $\mu \mathrm{m}$-order sized $\mathrm{Cu}$ with a branch-like shape, $\mu \mathrm{m}$ order sized $\mathrm{Ni}$ with a grain-like shape with many jags, and $10 \mathrm{~nm}$ spherical $\mathrm{Fe}_{3} \mathrm{O}_{4}$, because the $\mathrm{Cu}$, Ni particles and $\mathrm{MF}$ are compounded. In Figure 13(b), the particles in the MCF rubber are $1.2 \mu \mathrm{m}$ spherical $\mathrm{Fe}$ and $10 \mathrm{~nm}$ spherical $\mathrm{Fe}_{3} \mathrm{O}_{4}$, because the Fe particles and MF are compounded.

Figure 14 shows a comparison of electric resistance to compressed deformation between MCF rubber and commercial electric conductive rubber. The MCF rubber is $20 \mathrm{~mm} \times$ $15 \mathrm{~mm} \times 0.622 \mathrm{~mm}$, and the commercial electric conductive rubber is $30 \mathrm{~mm} \times 30 \mathrm{~mm} \times 0.455 \mathrm{~mm}$.

\section{References}

[1] K. Shimada, T. Fujita, H. Oka, Y. Akagami, and S. Kamiyama, "Hydrodynamic and magnetized characteristics of MCF (magnetic compound fluid)," Transactions of the Japan Society of Mechanical Engineers B, vol. 67, no. 664, pp. 3034-3040, 2001.

[2] K. Shimada, Y. Akagami, T. Fujita, T. Miyazaki, S. Kamiyama, and A. Shibayama, "Characteristics of magnetic compound fluid (MCF) in a rotating rheometer," Journal of Magnetism and Magnetic Materials, vol. 252, no. 1-3, pp. 235-237, 2002.

[3] K. Shimada, T. Fujita, S. Kamiyama, and Y. Akagami, "Experimental investigation of effects by various factors on shear flow characteristics of magnetic compound fluid (MCF) and its application," Journal of the Japan Society of Applied Electromagnetics and Mechanics, vol. 10, no. 1, pp. 67-72, 2002.

[4] T. Fujita and K. Shimada, "Characteristics and application of magnetorheological fluid," Recent Research Development Magnetism and Magnetic Materials, vol. 1, pp. 463-479, 2003.

[5] K. Shimada, T. Miyazaki, A. Shibayama, and T. Fujita, "Extraction of magnetic particle clusters self-assembled by a magnetic field," Smart Materials and Structures, vol. 12, no. 2, pp. 297-303, 2003. 
[6] K. Shimada, S. Shuchi, H. Kanno, Y. Wu, and S. Kamiyama, "Magnetic cluster and its applications," Journal of Magnetism and Magnetic Materials, vol. 289, pp. 9-12, 2005.

[7] K. Shimada, H. Kanno, J. Ogawa, S. Shuchi, and S. Kamiyama, "Development of non-sedimentation type damper," Transactions of the Japan Society of Mechanical Engineers B, vol. 69, no. 685, pp. 2075-2082, 2003.

[8] H. Kanno, K. Shimada, and J. Ogawa, "Experimental investigation of variable damping properties for viscous damper utilizing a magnetic responsive fluid," Transactions of the Japan Society of Mechanical Engineers B, vol. 71, no. 703, pp. 869876, 2005.

[9] H. Kanno, K. Shimada, J. Ogawa, and N. Inoue, "MR fluid damper composed of different size of particles," International Journal of Applied Electromagnetics and Mechanics, vol. 25, no. 1-4, pp. 109-112, 2007.

[10] Y. Wu, K. Shimada, Y. C. Wong, and M. Kato, "Effects of particles blend ratio on surface quality in surface polishing using Magnetic Polishing Liquid (MPL)," Key Engineering Materials, vol. 291-292, pp. 337-342, 2005.

[11] K. Shimada, Y. Matuo, K. Yamamoto, and Y. Zheng, "Study on new float polishing with the MCF," Journal of Achivements in Materials and Manufacturing Engineering, vol. 23, no. 2, pp. 91-94, 2007.

[12] K. Shimada, Y. Matuo, K. Yamamoto, and Y. Wu, "A new floatpolishing technique with large clearance utilizing magnetic compound fluid," International Journal of Abrasive Technology, vol. 1, no. 3-4, pp. 302-315, 2008.

[13] K. Shimada, S. Shuchi, and H. Kanno, "Magnetic rubber having magnetic clusters composed of metal particles," Journal of Intelligent Material Systems and Structures, vol. 16, no. 1, pp. 15-20, 2005.

[14] K. Shimada and Y. Zheng, "Basic study on tensile characteristics of MCF (magnetic compound fluid) rubber," Transactions of the Japan Society of Mechanical Engineers B, vol. 74, no. 11, pp. 2287-2294, 2008.

[15] K. Shimada and Y. Zheng, "Study on electric conductivity under tensile condition of MCF rubber for haptic robot sensor," Transactions of the Japan Society of Mechanical Engineers $B$, vol. 74, no. 8, pp. 1748-1754, 2008.

[16] M. Tanaka, J. Hiraizumi, J. L. Lévêque, and S. Chonan, "Haptic sensor for monitoring skin conditions," International Journal of Applied Electromagnetics and Mechanics, vol. 14, no. 1-4, pp. 397-404, 2001.

[17] R. Tajima, S. Kagami, M. Inaba, and H. Inoue, "Development of soft and distributed tactile sensors and the application to a humanoid robot," Advanced Robotics, vol. 16, no. 4, pp. 381397, 2002.

[18] K. Shimada and Y. Zheng, "Development of MCF rubber with temperature and electric senses for an elemnt material in haptic robot sensor," Transactions of the Japan Society of Mechanical Engineers B, vol. 73, no. 735, pp. 2269-2274, 2007.

[19] Y. Zheng and K. Shimada, "Study on a haptic sensor using MCF (magnetic compound fluid) electric conductive rubber," Journal of Solid Mechanics and Materials Engineering, vol. 2, no. 6, pp. $748-755,2008$.

[20] Y. Zheng and K. Shimada, "Research on a haptic sensor made using MCF conductive rubber," Journal of Physics Condensed Matter, vol. 20, no. 20, Article ID 204148, 2008.

[21] Y. Zheng and K. Shimada, "Basic study on trial production of haptic sensor utilizing MCF (magnetic compound fluid) electric conductive rubber," Jounal of Japan Society Experimental Mechanics, vol. 7, no. 3, pp. 234-239, 2007.
[22] Y. Zheng and K. Shimada, "Development of improved MCF rubber on MCF touch sensor," Journal of Japan Society Experimental Mechanics, vol. 8, no. 4, pp. 392-395, 2008.

[23] J. R. Pyne, "Conductive rubbers advance with new blacks," European Rubber Journal, pp. 17-20, November 1981.

[24] S. Wang and D. D. L. Chung, "Piezoresistivity in continuous carbon fiber polymer-matrix composite," Polymer Composites, vol. 21, no. 1, pp. 13-19, 2000.

[25] J. Cao, Q. Wang, and H. Dai, "Electromechanical properties of metallic, quasimetallic, and semiconducting carbon nanotubes under stretching," Physical Review Letters, vol. 90, no. 15, Article ID 157601, 4 pages, 2003.

[26] B. L. Pruitt, W. T. Park, and T. W. Kenny, "Measurement system for low force and small displacement contacts," Journal of Microelectromechanical Systems, vol. 13, no. 2, pp. 220-229, 2004.

[27] G. Ausanio, A. C. Barone, C. Campana et al., "Giant resistivity change induced by strain in a composite of conducting particles in an elastomer matrix," Sensors and Actuators A, vol. 127, no. 1, pp. 56-62, 2006.

[28] V. Maheshwari and R. F. Saraf, "High-resolution thin film device to sense texture by touch," Science, vol. 312 , no. 5779, pp. 1501-1504, 2006.

[29] A. Van Roggen, "Electronic conduction of polymer single crystals," Physical Review Letters, vol. 9, no. 9, pp. 368-370, 1962.

[30] R. D. Sherman, L. M. Middleman, and S. M. Jacobs, "Electron transport processes in conductor-filled polymers," Polymer Engineering and Science, vol. 23, no. 1, pp. 36-46, 1983.

[31] K. Shimamura, M. Hatano, S. Kanbara, and I. Nakada, "Electrical conduction of poly-acetylene under high pressure," Journal of Physical Society of Japan, vol. 23, no. 3, pp. 578-581, 1967.

[32] E. K. Sichel, J. I. Gittleman, and P. Sheng, "Transport properties of the composite material carbon-poly(vinyl chloride)," Physical Review B, vol. 18, no. 10, pp. 5712-5716, 1978.

[33] P. Sheng, "Fluctuation-induced tunneling conduction in disordered materials," Physical Review B, vol. 21, no. 6, pp. 21802195, 1980.

[34] S. Nishio, T. Takeuchi, Y. Matsuura, K. Yoshizawa, K. Tanaka, and T. Yamabe, "Electrical conductivity measurements of the plasma-polymerized 1-benzothiophene thin film," Synthetic Metals, vol. 46, no. 2, pp. 243-250, 1992.

[35] S. Banerjee and D. Chakravorty, "Alternating current conductivity and dielectric dispersion in copper-silica nanocomposites synthesized by electrodeposition," Journal of Applied Physics, vol. 84, no. 2, pp. 799-805, 1998.

[36] R. Gangopadhyay, A. De, and S. Das, “Transport properties of polypyrrole-ferric oxide conducting nanocomposites," Journal of Applied Physics, vol. 87, no. 5, pp. 2363-2371, 2000.

[37] S. Bhattacharya and A. Ghosh, "Electrical transport properties of semiconducting lithium molybdate glass nanocomposites," Journal of Chemical Physics, vol. 127, no. 19, Article ID 194709, 2007.

[38] A. Van Roggen and P. H. E. Meijer, "The effect of electrodepolymer interfacial layers on polymer conduction," IEEE Transactions on Electrical Insulation, vol. 21, no. 3, pp. 307311, 1985.

[39] K. Shigeo, T. Sotomura, Y. Yamamura, T. Kanbara, and K. Takada, "Electrochemical properties of paper form soild electrolyte," Technical Study Report of IEICE, vol. 87, no. 340, pp. 21-26, 1988. 
[40] P. H. E. Meijer and A. van Roggen, "Polymer electronic devices," Journal of Molecular Electronics, vol. 4, no. 2, pp. 119124, 1988.

[41] A. V. Roggen and P. H. E. Meijer, "The effect of electrodepolymer interfacial layers on polymer conduction, part 2: device summary," in Molecular Electronic Devices, F. L. Carter, R. E. Siatkowski, and H. Wohltjen, Eds., pp. 427-437, Elsevier/North-Holland, Amsterdam, The Netherlands, 1988.

[42] D. K. Ferry, Quantum Mechanics: An Introduction for Device Physics and Electrical Engineers, Springer, Tokyo, Japan, 2006, translated by Y. Ochiai, M. Uchinami, K. Matsuda and K. Ishibashi.

[43] M. Tanaka, J. Hiraizumi, J. L. Lévêque, and S. Chonan, "Haptic sensor for monitoring skin conditions," International Journal of Applied Electromagnetics and Mechanics, vol. 14, no. 1-4, pp. 397-404, 2001.

[44] R. Tajima, S. Kagami, M. Inaba, and H. Inoue, "Development of soft and distributed tactile sensors and the application to a humanoid robot," Advanced Robotics, vol. 16, no. 4, pp. 381397, 2002.

[45] Y. Shimizu, Forefront of Development of the Super Five Senses Sensor, T. Yoshida, et al., Eds., NTS, Japan, 2005. 

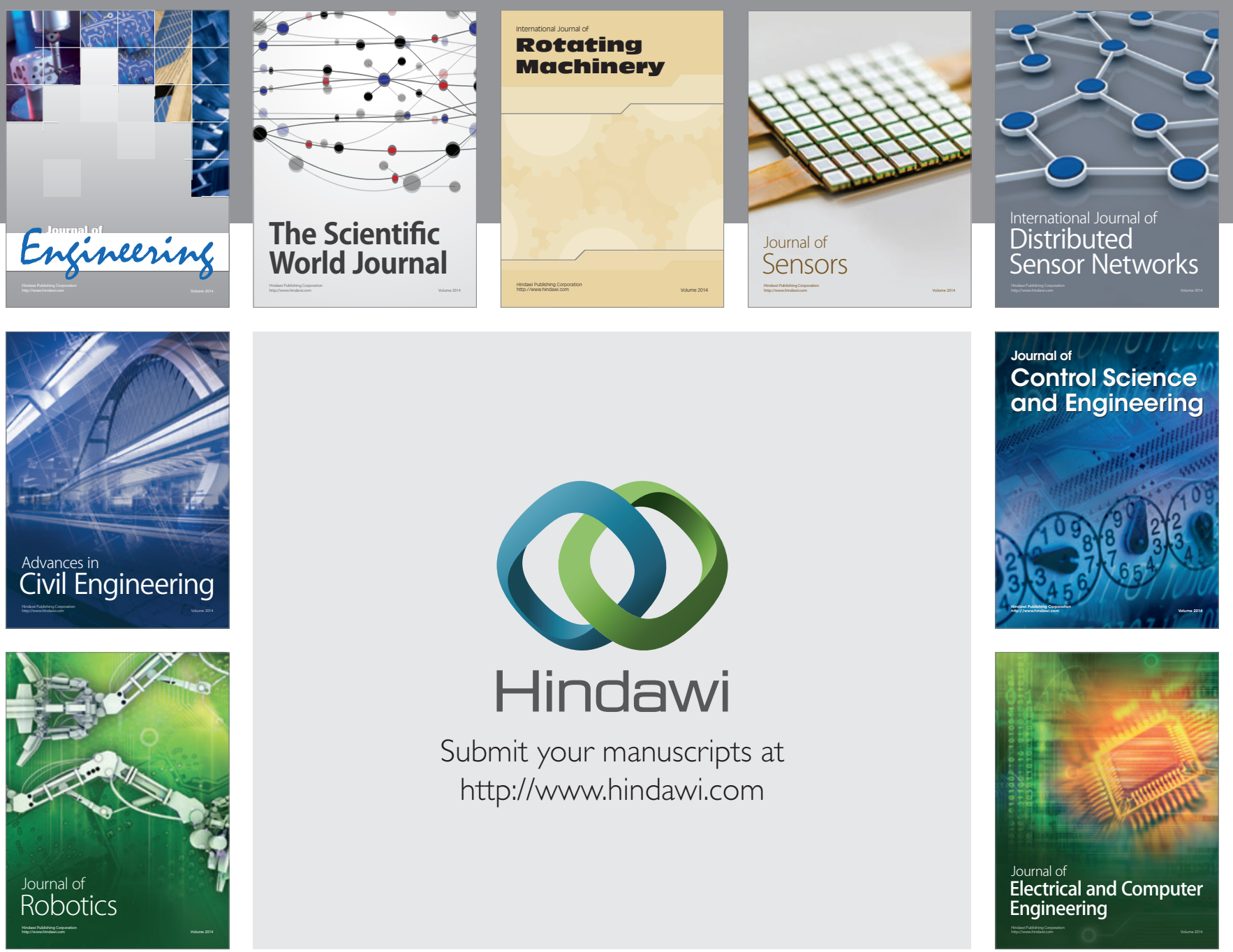

Submit your manuscripts at

http://www.hindawi.com
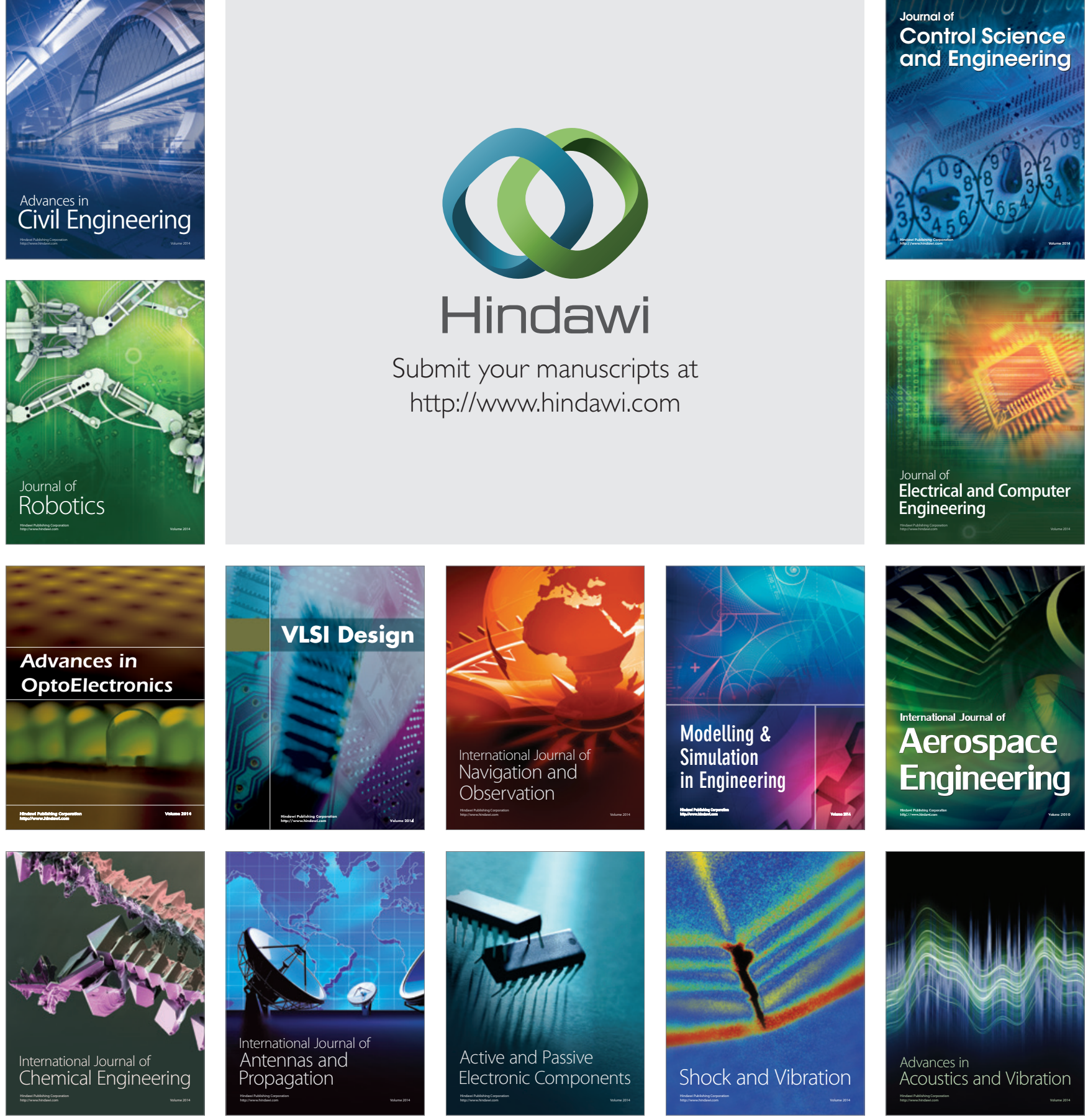